\title{
A Rare Presentation of Schwannoma as a Purely- Cystic Superficial-Intermuscular Extra-Spinal Swelling over Upper Back.
}

\section{Srinjoy Saha ( $\sim$ ss@medi.ac)}

Apollo Gleneagles Hospital Kolkata https://orcid.org/0000-0001-8971-148X

\section{Case Report}

Keywords: Adult, Female, Humans, Magnetic Resonance Imaging, Neurilemmoma / diagnosis, Neurilemmoma / pathology, Neurilemmoma / surgery, Peripheral Nervous System Neoplasms / diagnosis, Peripheral Nervous System Neoplasms / pathology, Peripheral Nervous System Neoplasms / surgery,

Posted Date: June 30th, 2020

DOI: https://doi.org/10.21203/rs.3.rs-38097/v1

License: (a) (i) This work is licensed under a Creative Commons Attribution 4.0 International License. Read Full License 


\section{Abstract}

Schwannomas are rare tumours arising from peripheral nerve sheath. Here, a 44-year-old lady came to our clinic with an asymptomatic progressively-enlarging swelling over her upper back. It was fluctuant, with an absence of pain and tenderness. MRI showed a benign, purely-cystic, superficial-intermuscular, extra-spinal swelling nearby upper thoracic vertebrae. Complete surgical excision proceeded smoothly through a well-defined plane between the swelling and the muscles. It was not attached to any identifiable nerve. A $6.5 \times 5.0 \times 2.5 \mathrm{~cm}$ ovoid lesion with a glistening whitish-grey capsule was excised and wound reconstructed in layers. Histopathology showed hypercellular areas with nuclear palisading or oval-shaped Verocay bodies. Only S-100 tested positive amongst the five-antigen immunohistochemistry, thus establishing the diagnosis of a schwannoma. Postoperatively, a one-year follow-up period was uneventful. Schwannomas can surprise clinicians by arising anywhere and with atypical presentations. It needs to be in the differential diagnoses of any asymptomatic slowly-growing lesion.

\section{Introduction}

Schwannomas (also called neurilemmomas) are tumours arising from the schwann cells, which sheathe, support and protect the peripheral nerves by warping around axons. Usually, they are benign encapsulated solid tumours, and rarely cancerous [1]. On magnetic resonance imaging (MRI), classical signs like 'split-fat sign' and 'target sign' may be present. Degenerative changes like hyalinisation, haemorrhage, calcification and cyst formation may occur [2]. Purely cystic schwannomas are rarely encountered. Described over here is an unusual presentation of an asymptomatic, purely-cystic, superficial-intermuscular, extra-spinal schwannoma over the upper back. It was not related to any known nerve course and presented without any classical diagnostic signs on MRI.

\section{Case Presentation}

A 44-year-old woman came to our clinic with an asymptomatic slowly progressive swelling over her upper back. She first noticed it four years ago. Her medical history was unremarkable, and she did not have any sensory or motor changes. Clinical examination revealed a spherical non-tender swelling in the paraspinal area over the upper back, sized $7.0 \times 6.5 \mathrm{~cm}$ [Figure 1]. Fluctuation was present on palpation, but thrill was absent. There were no other associated symptoms and no tenderness on spinal palpation. MRI revealed a well-defined, smooth-margin, oval-shaped swelling in the superficial-intermuscular plane. It lay underneath the trapezius, displacing the rhomboid and serratus posterior superior, and was superficial to the erector spinae muscles [Figure 2]. While extending deep and medially towards the right side of the spinous process, it stayed far-off the dorsal spinal rami. There was a clear plane between the cyst and surrounding muscles, with no local invasion [Figure 3]. Relationship with any identifiable nerve was absent, nor was it related to any nerve course. Central part appeared necrotic with blood-fluid levels, with calcifications in the wall [Figure 2A,3A]. Contrast-enhanced T1-weighted images showed ring-like capsular enhancement [Figures 2B,3B]. Fine needle aspiration cytology (FNAC) was inconclusive. The pre-operative 
diagnosis was that of a benign, purely-cystic, superficial-intermuscular, extra-spinal swelling, and the patient was scheduled for surgical excision.

After surgical exploration, incising the fascia and retracting the trapezius helped reach the cystic swelling underneath, covered with a glistening whitish-grey capsule [Figure 4]. It extended inferiorly and medially deep towards the spine, displacing the rhomboid and serratus posterior superior. Lying superficial to the erector spinae muscles, it extended towards the right spinous processes of upper thoracic vertebrae, but far-off from the spine or spinous ligaments [Figure 5]. Dissection proceeded effortlessly through a welldefined plane between the swelling and the surrounding muscles. While a fascial pedicle attached its base to the underlying soft tissues, it was not adherent to any surrounding nerves. After complete surgical excision, the resulting wound was reconstructed in layers from deep to superficial. Gross examination of the specimen revealed a cystic swelling sized $6.5 \times 5.0 \times 2.5 \mathrm{~cm}$ [Figure 6]. The cut-section contained dark reddish-brown areas, signifying haemorrhagic changes inside. Histopathological examination showed an encapsulated lesion with hyper and hypocellular areas. Bland oval to spindle-shaped cells was present. Hypercellular areas with nuclear palisading (oval-shaped Verocay bodies) were noted. Cystic degeneration was present in the centre. Neither nuclear atypia nor increased mitotic activity was visible [Figure 7]. Immunohistochemistry was positive for S-100, and negative for CD34, Cytokeratin-Pan (AE1/AE3), Smooth muscle actin, with Mib1 of 1\%. Final diagnosis confirmed benign cystic schwannoma. Recovery during the postoperative period was uneventful, and the wound healed well with minimal scarring. There were no recurrences or other complications during the next one-year follow-up.

\section{Discussion}

Neurogenic tumours comprise only about $5 \%$ of all benign soft-tissue tumours. Differential diagnoses of extra-spinal swellings include schwannoma, neurofibroma, meningioma, ependymoma, sarcoma, abscess, lymphoma, and osteoma amongst others [3]. Schwannomas are most commonly found intradurally. They may occur at any level of the spinal cord axis, including as a dumb-bell shaped tumour with both intra-dural and extra-dural components [4]. A purely-cystic, extra-spinal, superficial presentation has not been reported yet.

Amongst the common benign nerve sheath tumours, neurofibromas are located centrally and are inseparable from the originating nerve. In direct contradiction, schwannomas are located eccentrically over its originating nerve sheath [5]. Typical schwannomas are well-encapsulated and solid, with membranes composed of a thin fibrous capsule. Degenerations occur within long-standing tumours, including hyalinisation, haemorrhage, calcification and cyst formation [6]. Most commonly affected regions are head and neck $(25 \%-45 \%)$ with the lateral neck being the most affected region. Manipulation of the swelling sometimes produces paraesthesia or 'shocks' in the distribution of the affected nerve [7]. Greater mobility from side-to-side compared to longitudinal is found in benign neurogenic tumours.

MRI is useful for pre-operative diagnosis. It may show characteristic 'split-fat sign', wherein a rim of fat surrounds the lesion. 'Entry-and -exit' signs of nerve roots entering and exiting the tumour are commonly 
visible at both ends of the fusiform mass. 'Target' sign is seen when the lesion shows a thin peripheral hyperintense rim and a central lower-signal intensity on T2-weighted images. Signs of degeneration like cyst formation, haemorrhage (blood-fluid levels), and calcification may be identifiable [8].

Histological characteristics of schwannoma include Antoni A lesions, where broad interlacing ribbons of extended spindle cells with elongated nuclei are arranged in waves, drifts and whorls. On cross-section, these cylindrical cells produce a palisading pattern of nuclei around a central cytoplasmic mass called Verocay body. Antoni B pattern is loose tissues lacking the arrangement of bundles and palisades and is considered a degenerative form of Type A [9]. Mitoses are absent usually, and malignant transformation is exceptional. Immunohistochemistry with s-100 staining establishes the neural origin of the tumour [10].

\section{Conclusion}

Schwannoma can surprise clinicians by arising anywhere in the body. It may not be related to any known nerves on MRI or during surgical exploration. It may be considered within the differential diagnoses of any slow-growing (including cystic) swelling. Careful surgical dissection while identifying and avoiding any nerve transection during surgical excision is necessary. Histopathology confirms, and immunohistochemistry establishes the diagnosis. Complete surgical removal leads to good postoperative prognosis.

\section{Declarations}

Approval: As this is a case report without identifiers, our institution does not require approval from the Institutional Review Board (IRB) or its equivalent.

Consent: The patient's consent for publication was obtained in writing.

Acknowledgments: Dr Sanjib Majumdar, MD; Dr Rajesh Ghosh, MD; and Dr Asra Nausheen Quadri, MD.

Disclosure: Dr Srinjoy Saha is an unpaid consultant with Dr Reddy's Laboratories, India. The scope of such involvement is not relevant to the current field of study.

\section{References}

1. Kim DH, Murovic JA, Tiel RL, Moes G, Kline DG. A series of 397 peripheral neural sheath tumors: 30 year experience at Louisiana State University Health Sciences Center. J Neurosurg. 2005; 102(2): 246-255.

2. Crist J, Hodge JR, Frick M, et al. Magnetic Resonance Imaging Appearance of Schwannomas from Head to Toe: A Pictorial Review. J Clin Imaging Sci. 2017; 7:38. 
3. Kransdorf $\mathrm{M}$ J. Benign soft-tissue tumors in a large referral population: distribution of specific diagnoses by age, sex, and location. American Journal of Roentgenology. 1995; 164:2, 395-402

4. Montano N, D'Alessandris QG, D'Ercole M, et al. Tumors of the peripheral nervous system: analysis of prognostic factors in a series with long-term follow-up and review of the literature. J Neurosurg. 2016; 125(2): 363-371.

5. MacCollin M, Chiocca EA, Evans DG, et al. Diagnostic criteria for schwannomatosis. Neurology. 2005; 64(11): 1838-1845

6. Knight DM, Birch R, Pringle J. Benign solitary schwannomas: a review of 234 cases. J Bone Joint Surg Br 2007; 89: 382-387

7. Farschtschi SC, Mainka T, Glatzel M, et al. C-Fiber Loss as a Possible Cause of Neuropathic Pain in Schwannomatosis. Int J Mol Sci. 2020; 21(10): 3569.

8. Zhang Z, Deng L, Ding L, Meng Q. MR imaging differentiation of malignant soft tissue tumors from peripheral schwannomas with large size and heterogeneous signal intensity. Eur J Radiol. 2015; 84(5): 940-946.

9. Goldblum, J.R., Folpe, A. L., Weiss, S.W., Enzinger and Weiss's Soft Tissue Tumors. pp. 813-829. 6th ed 2014. Philadelphia, PA: Mosby Elsevier.

10. Louis DN, Ohgaki H, Wiestler OD, Cavenee WK, Ellison DW, Branger FD, et al., editors. WHO Classification of Tumours of the Central Nervous System. 4th ed., Revised. Lyon: International Agency for Research Centre; 2016.

\section{Figures}




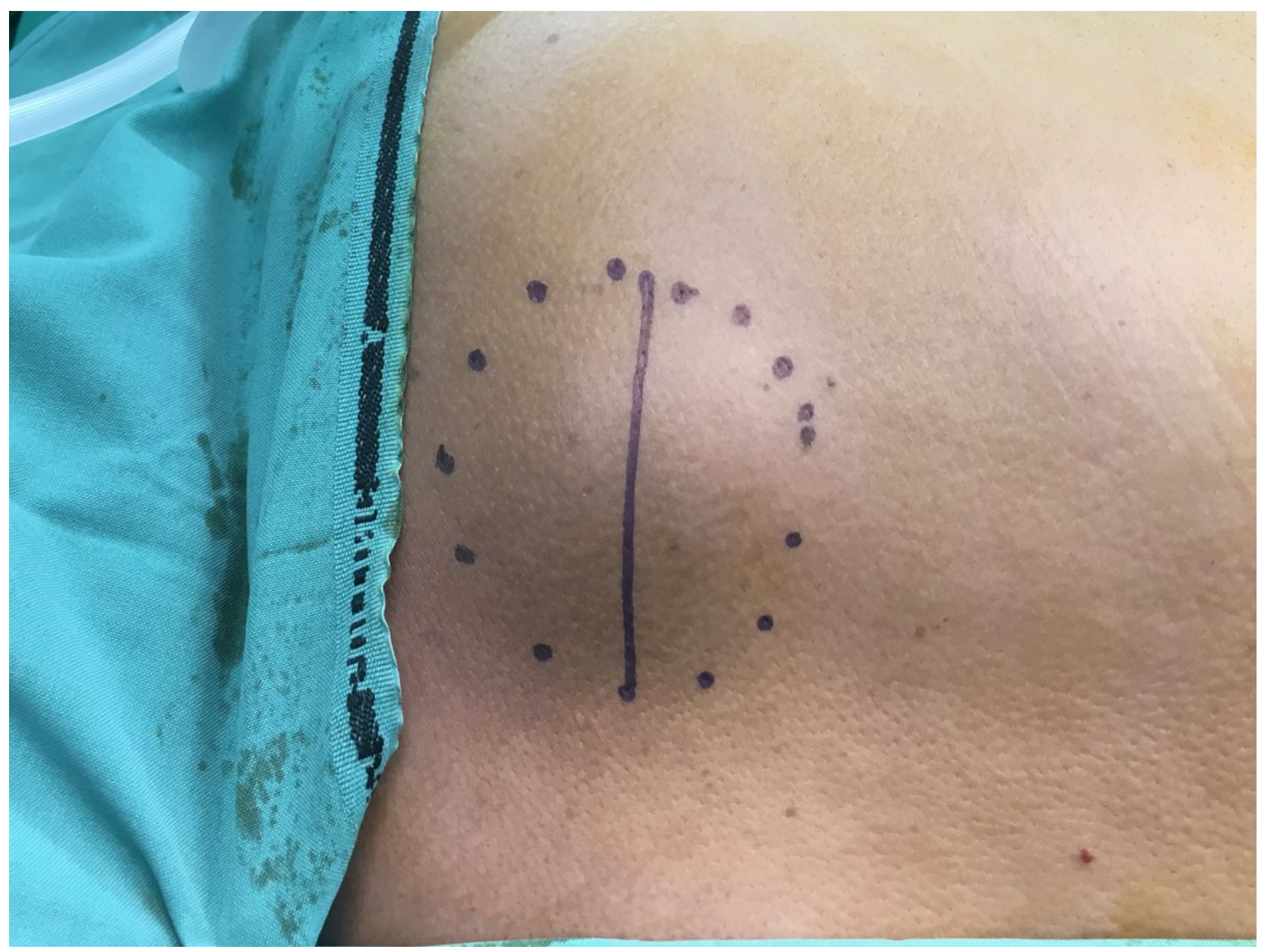

\section{Figure 1}

The fluctuant swelling over the upper back measured $7.0 \times 6.5 \mathrm{~cm}$ 


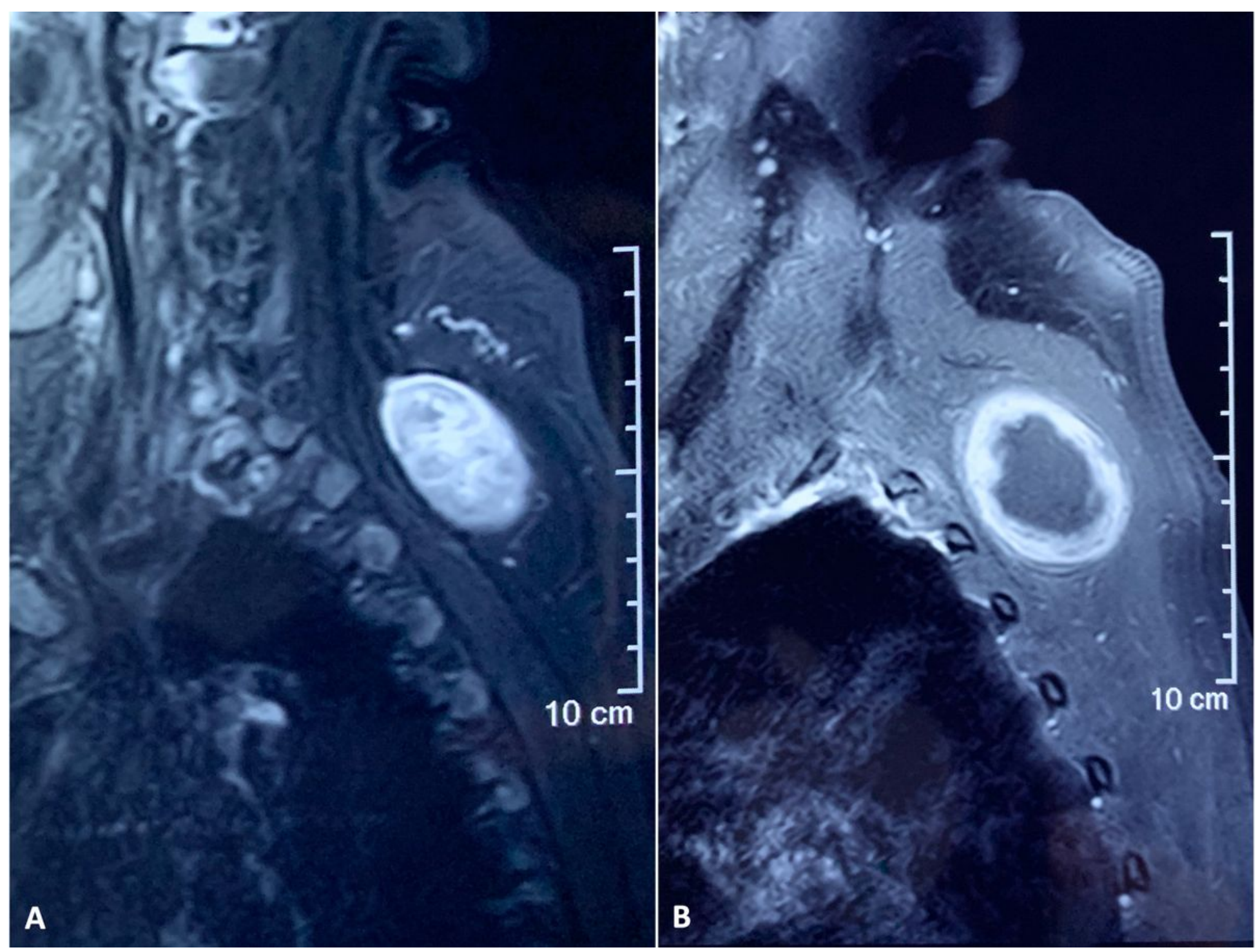

Figure 2

(A) MRI shows the lesion lying superficial to the erector spinae muscle in the region of the upper thoracic vertebrae. (B) T1-weighted image with rim-enhancement, signifying a purely cystic swelling. 


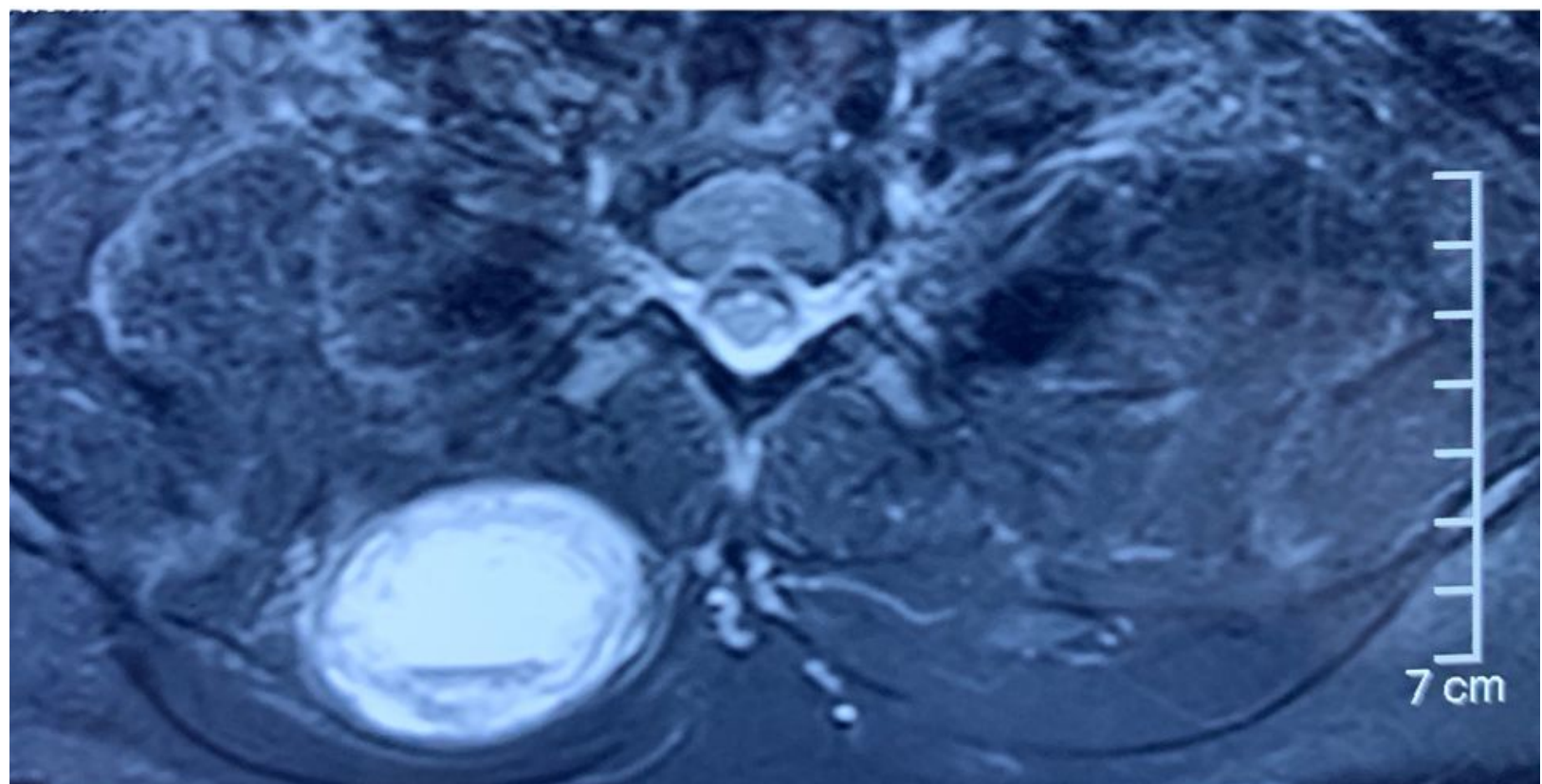

A

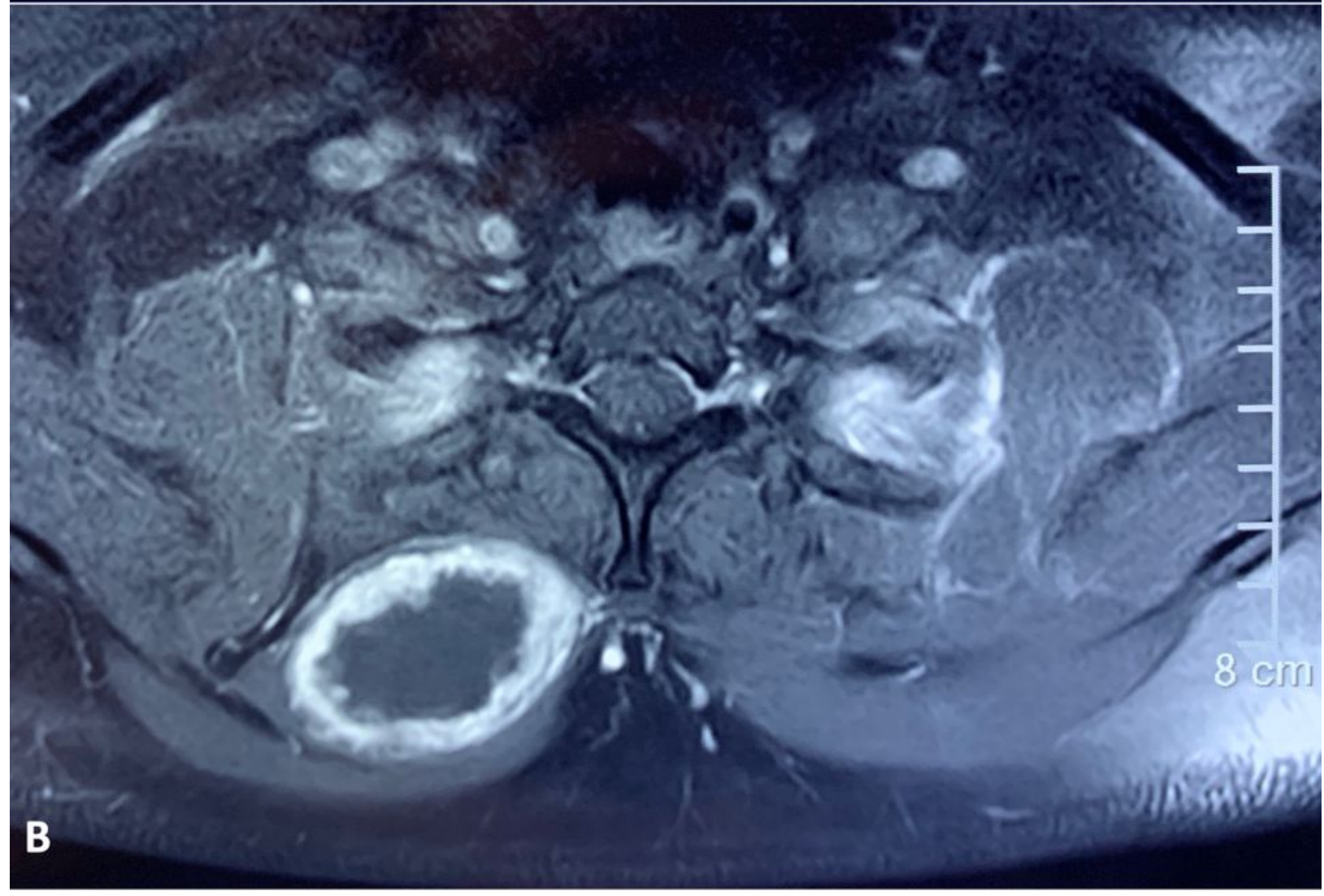

Figure 3

(A) MRI showed the lesion to be far-off from the spine, in an intermuscular plane. (B) T1-weighted image shows rim-enhancement of the lesion and displacement of some back muscles. 


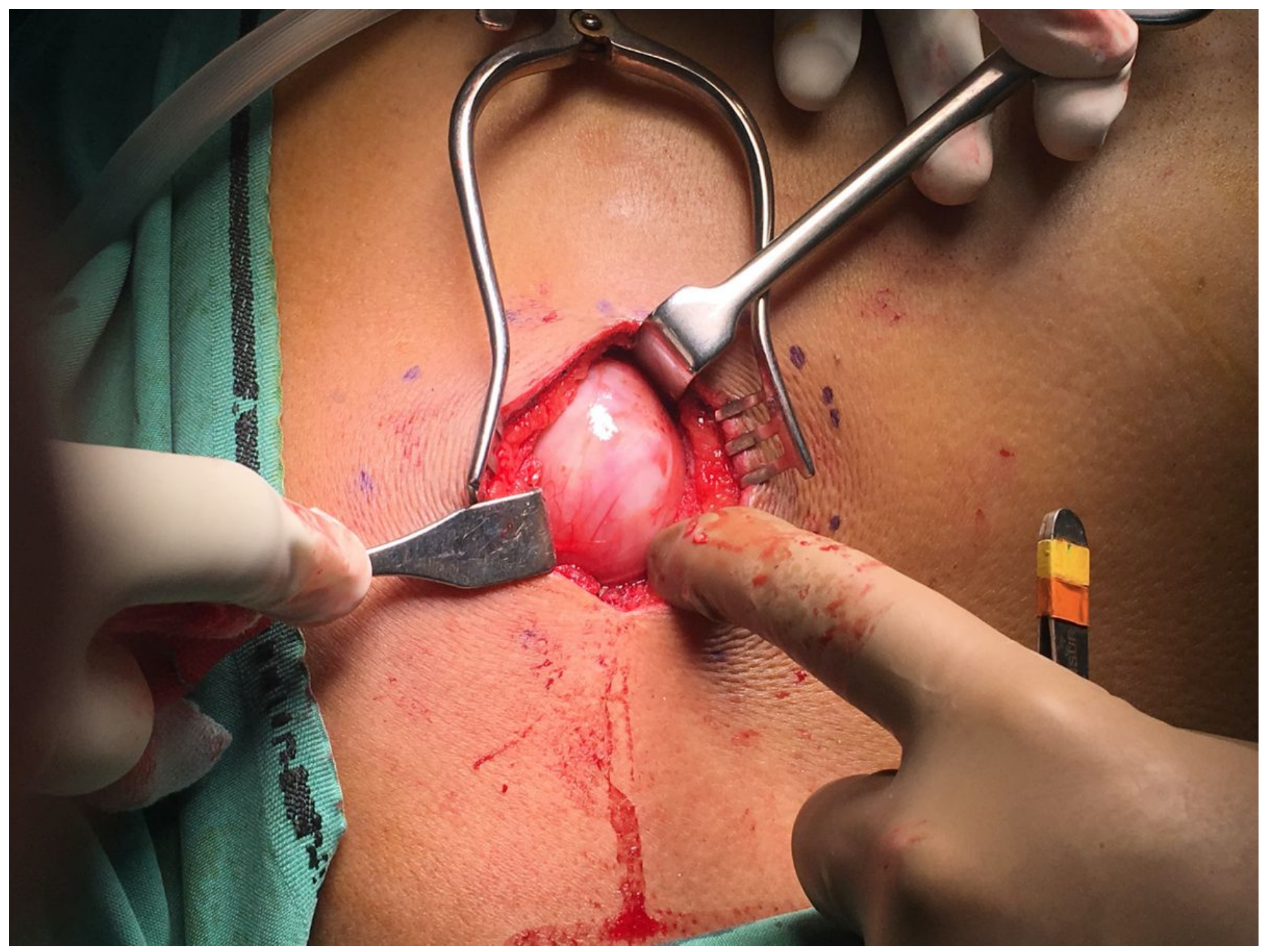

Figure 4

The fascia was incised, and trapezius retracted to reach the swelling covered with a glistening whitish capsule. 


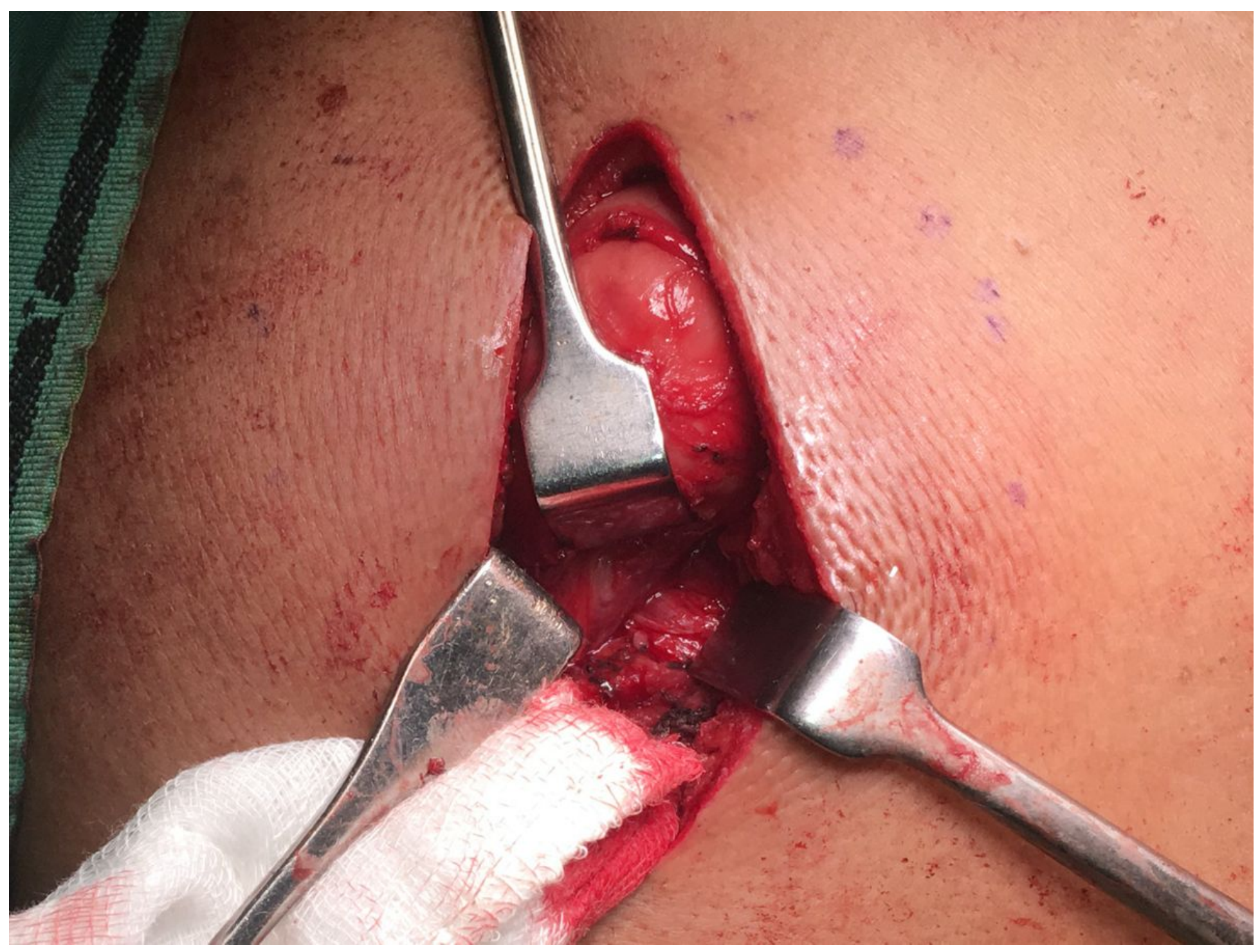

Figure 5

The swelling was far-off from the spinous processes and spinous ligaments, and not attached to any specific nerve. 


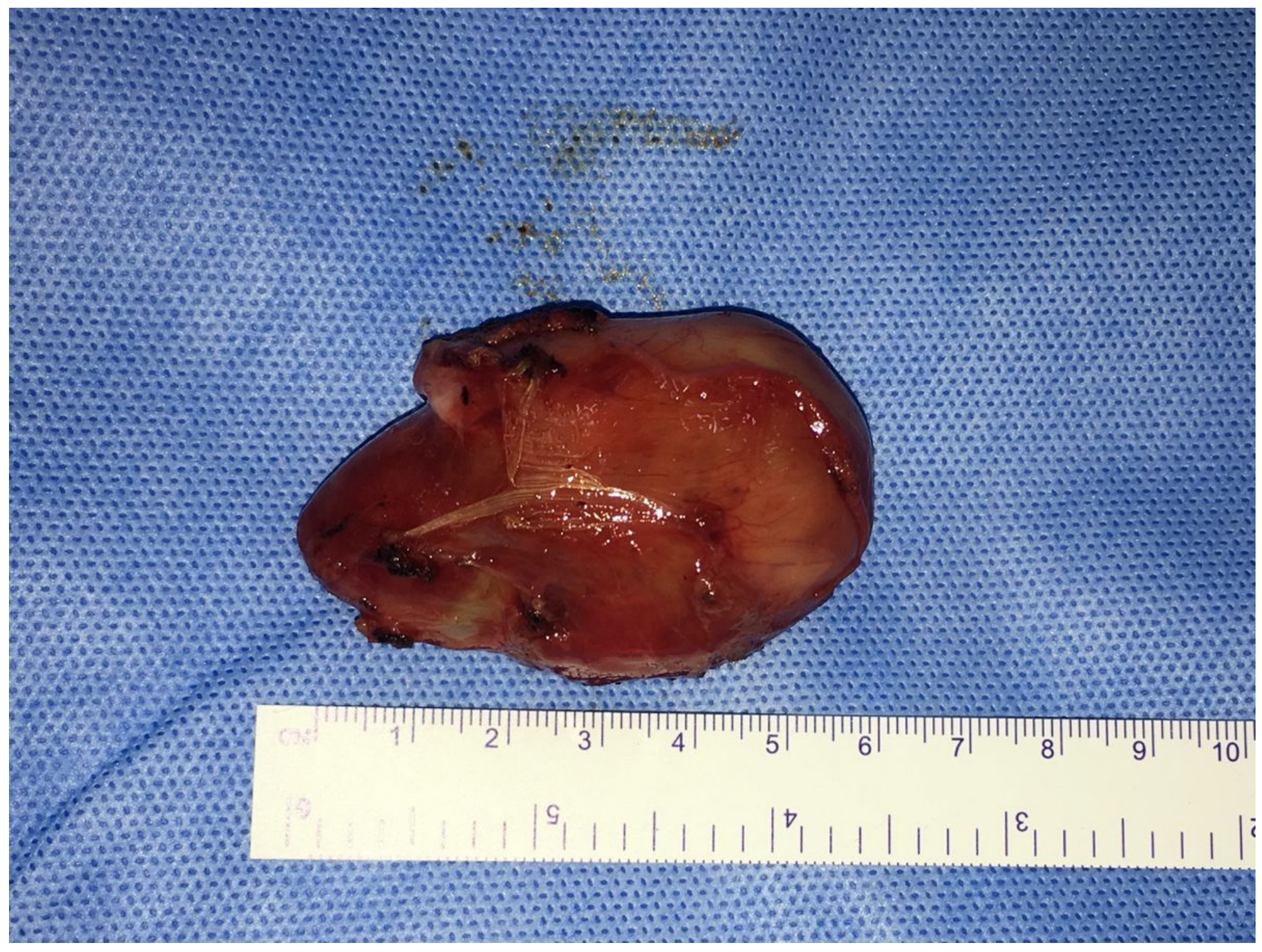

Figure 6

Complete excision revealed a cystic swelling sized $6.0 \times 5.0 \times 2.5 \mathrm{~cm}$. 


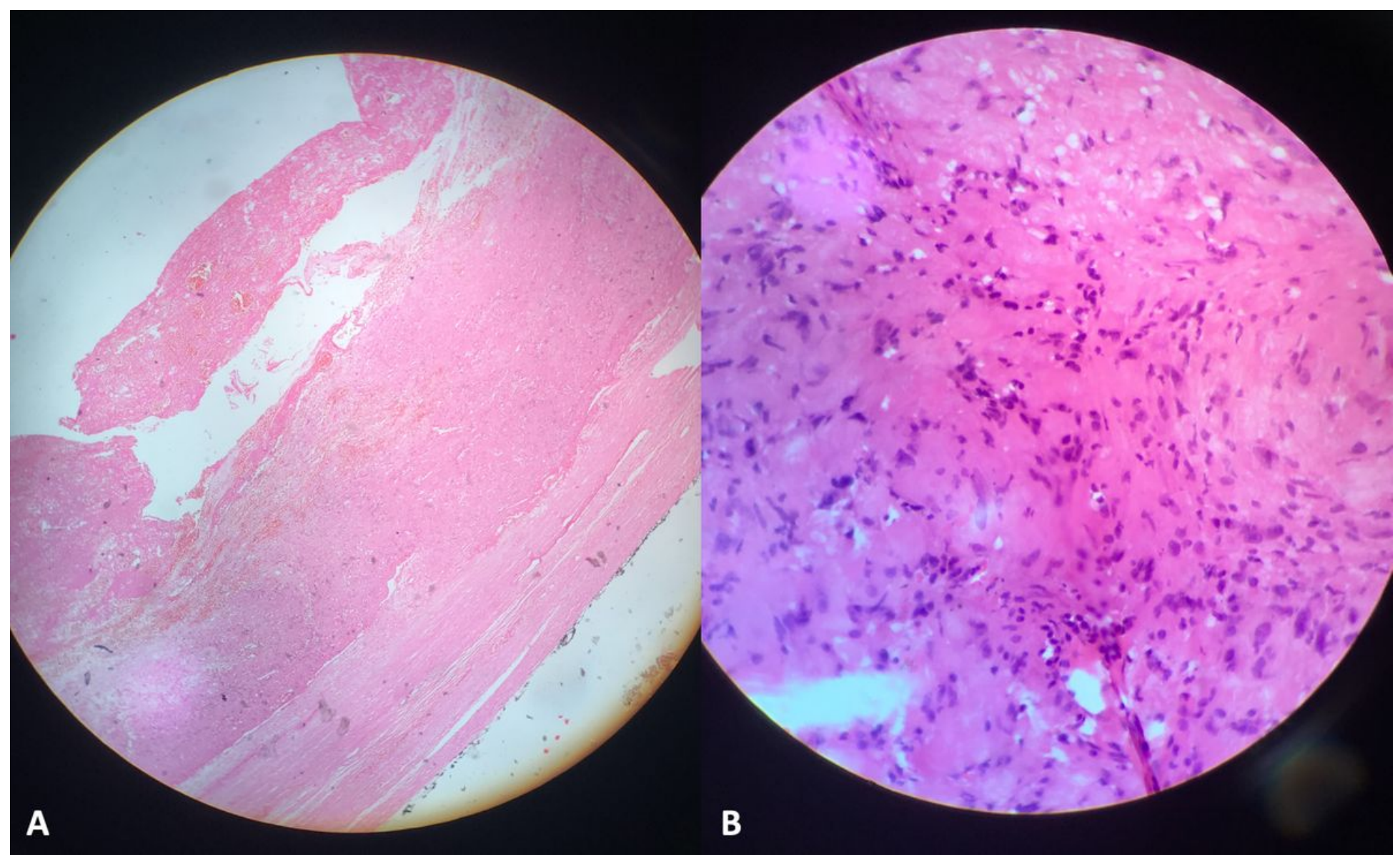

Figure 7

(A) Hematoxylin \& Eosin staining of the specimen shows the wall of the cystic swelling under scanner view. (B) Oval-shaped Verocay bodies, with characteristic nuclear palisading, was identifiable on highpower fields. 